\title{
Control Chaos in System with Fractional Order
}

\author{
Yamin Wang ${ }^{1}$, Xiaozhou Yin ${ }^{2}$, Yong Liu ${ }^{3 *}$ \\ ${ }^{1}$ Basis Course of Lianyungang Technical College, Lianyungang, China \\ ${ }^{2}$ Lianyungang Technical College, Lianyungang, China \\ ${ }^{3}$ School of Mathematical Science, Yancheng Teachers University, Yancheng, China \\ Email: ${ }^{*}$ yongliumath@163.com
}

Received February 26, 2012; revised March 18, 2012; accepted March 28, 2012

\begin{abstract}
In this paper, by utilizing the fractional calculus theory and computer simulations, dynamics of the fractional order system is studied. Further, we have extended the nonlinear feedback control in ODE systems to fractional order systems, in order to eliminate the chaotic behavior. The results are proved analytically by stability condition for fractional order system. Moreover numerical simulations are shown to verify the effectiveness of the proposed control scheme.
\end{abstract}

Keywords: Chaos; Fractional Order System; Nonlinear Feedback Control

\section{Introduction}

Fractional calculus is a classical mathematical concept, with a history as long as calculus itself. It is a generalization of ordinary differentiation and integration to arbitrary order, and is the fundamental theories of fractional order dynamical systems. Fractional-order differential/integral has been applied in physics and engineering, such as viscoelastic system [1], dielectric polarization [2], electrode-electrolyte polarization [3] and electromagnetic wave [4], and so on.

The fractional order system and its potential application in engineering field become promising and attractive due to the development of the fractional order calculus. Typically, chaotic systems remain chaotic when their equations become fractional. For example, it has been shown that the fractional order Chua's circuit with an appropriate cubic nonlinearity and with an order as low as 2.7 can produce a chaotic attractor [5].

However, there are essential differences between ordinary differential equation systems and fractional order differential systems. Most properties and conclusions of ordinary differential equation systems cannot be extended to that of the fractional order differential systems. Therefore, the fractional order systems have been paid more attention. Recently, many investigations were devoted to the chaotic dynamics and chaotic control of fractional order systems [6-12].

In this paper, practical scheme is proposed to eliminate the chaotic behaviors in fractional order system by extending the nonlinear feedback control in ODE systems to fractional-order systems. This paper is organized as

\footnotetext{
${ }^{*}$ Corresponding author.
}

follows. In Section 2, the numerical algorithm for the fractional order system is briefly introduced. In Section 3, Dynamics of the fractional order system is numerically studied. In Section 4, general approach to feedback control scheme is given, and then we have extended this control scheme to fractional order system, numerical results are shown. Finally, in Section 5, concluding comments are given.

\section{Fractional Derivative and Numerical Algorithm}

There are two approximation methods for solving fractional differential equations. The first one is an improved version of the Adams-Bashforth-Moulton algorithm, and the rest one is the frequency domain approximation. The Caputo derivative definition involves a time-domain computation in which nonhomogenous initial conditions are needed, and those values are readily determined. In this paper, the Caputo fractional derivative defined in [13] is often described by

$$
D^{q} f(t)=J^{n-q} f^{(n)}(t), q>0,
$$

when $n$ is the first integer that is not less than $q, J^{\alpha}$ is the $\alpha$-order Riemann-Liouville integral operator which defined by

$$
J^{\alpha} f(t)=\frac{1}{\Gamma(\alpha)} \int_{0}^{t}(t-\tau)^{\alpha-1} f(\tau) \mathrm{d} \tau,
$$

where $\Gamma$ is the Gamma function, $0<\alpha \leq 1$.

Now we consider the fractional order system [14] which is given by 


$$
\left\{\begin{array}{l}
\frac{\mathrm{d}^{q_{1}} x}{\mathrm{~d} t^{q_{1}}}=-\mu x+y z \\
\frac{\mathrm{d}^{q_{2}} y}{\mathrm{~d} t^{q_{2}}}=-\mu y+(z-a) x, \\
\frac{\mathrm{d}^{q_{3}} z}{\mathrm{~d} t^{q_{3}}}=z+x y
\end{array}\right.
$$

where $q_{i}$ is the fractional order, $0<q_{i} \leq 1,(i=1,2,3)$.

By exploiting the Adams-Bashforth-Moulton scheme [15], the fractional order system (1) can be discretized as followings:

$$
\begin{aligned}
& x_{n+1}=x_{0}+\frac{h^{q_{1}}}{\Gamma\left(q_{1}+2\right)}\left(-\mu x_{n+1}^{\rho}+y_{n+1}^{\rho} z_{n+1}^{\rho}\right) \\
& +\frac{h^{q_{1}}}{\Gamma\left(q_{1}+2\right)} \sum_{j=0}^{n} \beta_{1, j, n+1}\left(-\mu x_{j}+y_{j} z_{j}\right), \\
& y_{n+1}=y_{0}+\frac{h^{q_{2}}}{\Gamma\left(q_{2}+2\right)}\left(-\mu y_{n+1}^{\rho}+\left(z_{n+1}^{\rho}-a\right) x_{n+1}^{\rho}\right) \\
& +\frac{h^{q_{2}}}{\Gamma\left(q_{2}+2\right)} \sum_{j=0}^{n} \beta_{2, j, n+1}\left(-\mu y_{j}+\left(z_{j}-a\right) x_{j}\right), \\
& z_{n+1}=z_{0}+\frac{h^{q_{3}}}{\Gamma\left(q_{3}+2\right)}\left(z_{n+1}^{\rho}+x_{n+1}^{\rho} y_{n+1}^{\rho}\right) \\
& +\frac{h^{q_{3}}}{\Gamma\left(q_{3}+2\right)} \sum_{j=0}^{n} \beta_{3, j, n+1}\left(z_{j}+x_{j} y_{j}\right) \text {, } \\
& x_{n+1}^{\rho}=x_{0}+\frac{1}{\Gamma\left(q_{1}\right)} \sum_{j=0}^{n} \gamma_{1, j, n+1}\left(-\mu x_{j}+y_{j} z_{j}\right) \text {, } \\
& y_{n+1}^{\rho}=y_{0}+\frac{1}{\Gamma\left(q_{2}\right)} \sum_{j=0}^{n} \gamma_{2, j, n+1}\left(-\mu y_{j}+\left(z_{j}-a\right) x_{j}\right) \text {, } \\
& z_{n+1}^{\rho}=z_{0}+\frac{1}{\Gamma\left(q_{3}\right)} \sum_{j=0}^{n} \gamma_{3, j, n+1}\left(z_{j}+x_{j} y_{j}\right), \\
& \beta_{i, j, n+1}= \\
& \begin{cases}n^{q_{i}+1}-\left(n-q_{i}\right)(n+1)^{q_{i}} & j=0, \\
(n-j+2)^{q_{i}+1}+(n-j)^{q_{i}+1}-2(n-j+1)^{q_{i}+1} & 1 \leq j \leq n, \\
1 & j=n+1,\end{cases} \\
& \gamma_{i, j, n+1}=\frac{h^{q_{i}}}{q_{i}}\left((n-j+1)^{q_{i}}-(n-j)^{q_{i}}\right), \\
& 0 \leq j \leq n, i=1,2,3 \text {. }
\end{aligned}
$$

\section{Dynamic Analysis of the Fractional Order System}

Theorem 1: The fractional linear autonomous system

$$
\begin{gathered}
D^{q} X=A X \\
X(0)=X_{0}
\end{gathered}
$$

is locally asymptotically stable if and only if

$$
\min _{i}\left|\arg \left(\lambda_{i}\right)\right|>\frac{\pi q}{2}, i=1,2, \cdots, n .
$$

Theorem 2: Suppose $x=x^{*}$ be an equilibrium point of a fractional nonlinear system

$$
D^{q} x=f(x)
$$

If the eigenvalues of the Jacobian matrix $A=\left.\frac{\partial f}{\partial x}\right|_{x=x^{*}}$ satisfy

$$
\min _{i}\left|\arg \left(\lambda_{i}\right)\right|>\frac{\pi q}{2}, i=1,2, \cdots, n,
$$

then the system is locally asymptotically stable at the equilibrium point $x=x^{*}$.

The system (1) has five equilibrium points:

$$
\begin{aligned}
& S_{0}=(0,0,0), S_{1}=\left(\frac{\beta}{\sqrt{\mu}}, \sqrt{\mu}, \beta\right), S_{2}=\left(-\frac{\beta}{\sqrt{\mu}},-\sqrt{\mu}, \beta\right), \\
& S_{3}=\left(\frac{\gamma}{\sqrt{\mu}}, \sqrt{\mu}, \gamma\right), S_{4}=\left(-\frac{\gamma}{\sqrt{\mu}},-\sqrt{\mu}, \gamma\right) . \\
& \text { where } \beta=\frac{a+\sqrt{a^{2}+4 \mu^{2}}}{2}, \gamma=\frac{a-\sqrt{a^{2}+4 \mu^{2}}}{2} .
\end{aligned}
$$

When $\mu=2, a=5$, we obtain

$$
\begin{aligned}
& S_{0}=(0,0,0), S_{1}=(4.0317,1.4142,5.7016), \\
& S_{2}=(-4.0317,-1.4142,5.7016), \\
& S_{3}=(-0.4961,1.4142,-0.7016), \\
& S_{4}=(0.4961,-1.4142,-0.7016) .
\end{aligned}
$$

First, we choose $S_{0}=(0,0,0)$ to study, the eigenvalues of the Jacobian matrix are $\lambda_{1}=\lambda_{2}=-2$ and $\lambda_{3}=1$. We can obtain $\arg \left(\lambda_{1}\right)=\arg \left(\lambda_{2}\right)=\pi$ and $\arg \left(\lambda_{3}\right)=0$. According to Theorem 2, we can easily conclude that the equilibrium $S_{0}$ of system (1) is unstable when $q_{1}, q_{2}$ and $q_{3}$ are all greater than zero.

We choose $S_{1}=(4.0317,1.4142,5.7016)$ and $S_{2}=(-4.0317,-1.4142,5.7016)$ to study, the eigenvalues of the Jacobian matrix are $\lambda_{1}=-4.0000, \lambda_{2}=0.50000+4.24316 i$ and $\lambda_{3}=0.50000-4.24316 i$. We can obtain $\arg \left(\lambda_{1}\right)=\pi, \arg \left(\lambda_{2}\right)=1.4535$ and $\arg \left(\lambda_{3}\right)=-1.4535$. According to Theorem 2, we can easily conclude that when $q_{1}, q_{2}$ and $q_{3}$ are all less than $0.9253 \approx 1.4535 \times \frac{2}{\pi}$, the equilibrium $S_{1}, S_{2}$ of system (1) is stable. On the contrary, when $q_{1}, q_{2}$ and 
$q_{3}$ are all great than 0.9253 , the equilibrium $S_{1}, S_{2}$ of system (1) is unstable.

Finally, when choose

$S_{3}=(-0.4961,1.4142,-0.7016)$ and

$S_{4}=(0.4961,-1.4142,-0.7016)$ to study, the eigenvalues of the Jacobian matrix are

$\lambda_{1}=-4.0000, \lambda_{2}=0.50000+1.41284 i$ and

$\lambda_{3}=0.50000-1.41284 i$. We can obtain

$\arg \left(\lambda_{1}\right)=\pi, \arg \left(\lambda_{2}\right)=1.2306$ and

$\arg \left(\lambda_{3}\right)=-1.2306$. According to Theorem 2, we can easily conclude that when $q_{1}, q_{2}$ and $q_{3}$ are all great than 0.7834 , the equilibrium $S_{3}, S_{4}$ of system (1) is unstable.

In sum, there exists at least one stable equilibrium $S_{1}, S_{2}, S_{3}$ and $S_{4}$ of system (1), when $q_{1}, q_{2}$ and $q_{3}$ are all less than 0.7834 , i.e., the system (1) will be stabilized at one point $\left(S_{1}, S_{2}, S_{3}\right.$ or $\left.S_{4}\right)$ finally; when $q_{1}, q_{2}$ and $q_{3}$ are all greater than 0.9253 , all the equilibriums of system (1) are unstable, the system (1) will exhibit a chaotic behaviour; when

$q_{i} \in(0.7834,0.9253)$, the problem will be complicated, the system (1) may be convergent, periodic or chaotic. For example, when $q_{1}=q_{2}=q_{3}=0.911$, the value of the largest Lyapunov exponent is 0.1653 . Obviously, the fractional order system (1) is chaotic. When

$q_{1}=q_{2}=q_{3}=0.91$, the fractional order system (1) is not chaotic, but periodic orbits appear.

\section{Feedback Control}

Let us consider the fractional order system

$$
D^{q} x(t)=f(x, u, t),
$$

where $x(t)$ is the system state vector, and $u(t)$ the control input vector. Given a reference signal $\tilde{x}(t)$, the problem is to design a controller in the state feedback form:

$$
u(t)=g(x, t),
$$

where $g(x, t)$ is the vector-valued function, so that the controlled system

$$
D^{q} x(t)=f(x, g(x, t), t)
$$

can be driven by the feedback control $g(x, t)$ to achieve the goal of target tracking so we must have

$$
\lim _{t \rightarrow t_{f}}\|x(t)-\tilde{x}(t)\|=0 .
$$

Let $\tilde{x}(t)$ be a periodic orbit or fixed point of the given system (2) with $u=0$, then we obtain the system error

$$
D^{q} e(t)=F(e, t)
$$

where $e=x-\tilde{x}$ and

$$
F(e, t)=f(x, g(x, t), t)-f(\tilde{x}, 0, t) \text {. }
$$

Theorem 3: If 0 is a fixed point of the system (2) and the eigenvalues of the Jacobian matrix at the equilibrium point 0 satisfies the condition

$$
\min _{i}\left|\arg \left(\lambda_{i}\right)\right|>\frac{\pi q}{2}, i=1,2, \cdots, n,
$$

then the trajectory $x(t)$ of system (2) converge to $\tilde{x}(t)$.

Let us consider the fractional order system (2), we propose to stabilize unstable periodic orbit or fixed point, the controlled system is as follows:

$$
\left\{\begin{array}{l}
\frac{\mathrm{d}^{q_{1}} x}{\mathrm{~d} t^{q_{1}}}=-\mu x+y z+u_{1}, \\
\frac{\mathrm{d}^{q_{2}} y}{\mathrm{~d} t^{q_{2}}}=-\mu y+(z-a) x+u_{2}, \\
\frac{\mathrm{d}^{q_{3}} z}{\mathrm{~d} t^{q_{3}}}=z+x y+u_{3},
\end{array}\right.
$$

Since $(\tilde{x}, \tilde{y}, \tilde{z})$ is solution of system (1), then we have:

$$
\left\{\begin{array}{l}
\frac{\mathrm{d}^{q_{1}} \tilde{x}}{\mathrm{~d} t^{q_{1}}}=-\mu \tilde{x}+\tilde{y} \tilde{z}, \\
\frac{\mathrm{d}^{q_{2}} \tilde{y}}{\mathrm{~d} t^{q_{2}}}=-\mu \tilde{y}+(\tilde{z}-a) \tilde{x}, \\
\frac{\mathrm{d}^{q_{3}} \tilde{z}}{\mathrm{~d} t^{q_{3}}}=\tilde{z}+\tilde{x} \tilde{y} .
\end{array}\right.
$$

Subtracting (4) from (3) with notation, $e_{1}=x-\tilde{x}, e_{2}=y-\tilde{y}, e_{3}=z-\tilde{z}$, we obtain the system error

$$
\left\{\begin{array}{l}
\frac{\mathrm{d}^{q_{1}} e_{1}}{\mathrm{~d} t^{q_{1}}}=-\mu e_{1}+y z-\tilde{y} \tilde{z}+u_{1}, \\
\frac{\mathrm{d}^{q_{2}} e_{2}}{\mathrm{~d} t^{q_{2}}}=-\mu e_{2}-a e_{1}+z x-\tilde{z} \tilde{x}+u_{2}, \\
\frac{\mathrm{d}^{q_{3}} e_{3}}{\mathrm{~d} t^{q_{3}}}=e_{3}-x y+\tilde{x} \tilde{y}+u_{3} .
\end{array}\right.
$$

We define the control function as follow

$$
\left\{\begin{array}{l}
u_{1}=-y z+\tilde{y} \tilde{z}, \\
u_{2}=-z x+\tilde{z} \tilde{x}, \\
u_{3}=x y-\tilde{x} \tilde{y}-\mu e_{3} .
\end{array}\right.
$$

So the system error (5) becomes

$$
\left\{\begin{array}{l}
\frac{\mathrm{d}^{q_{1}} e_{1}}{\mathrm{~d} t^{q_{1}}}=-\mu e_{1}, \\
\frac{\mathrm{d}^{q_{2}} e_{2}}{\mathrm{~d} t^{q_{2}}}=-\mu e_{2}-a e_{1}, \\
\frac{\mathrm{d}^{q_{3}} e_{3}}{\mathrm{~d} t^{q_{3}}}=(1-\mu) e_{3} .
\end{array}\right.
$$


The Jacobian matrix of system (7) is

$$
\left(\begin{array}{ccc}
-\mu & 0 & 0 \\
-a & -\mu & 0 \\
0 & 0 & 1-\mu
\end{array}\right)
$$

so we have the eigenvalues $\lambda_{1}=\lambda_{2}=-\mu$ and $\lambda_{3}=1-\mu$. When $\mu>1$ all eigenvalues are real negatives, one has $\arg \left(\lambda_{i}\right)=\pi$, therefore

$\left|\arg \left(\lambda_{i}\right)\right|>\frac{\pi q_{i}}{2}, i=1,2,3$, for all $q_{i}$ satisfies

$0<q_{i}<2, i=1,2,3$, it follows from Theorem 3 that the trajectory $x(t)$ of system (2) converges to $\tilde{x}(t)$ and the control is completed.

\section{Numerical Simulation}

In this section we give numerical results which prove the performance of the proposed scheme. As mentioned in Section 2 we have implemented the improved AdamsBashforth-Moulton algorithm for numerical simulation.
The control can be started at any time according to our needs, so we choose to activate the control when $t \geq 20$, in order to make a comparison between the behavior before activation of control and after it.

For $q_{1}=0.93, q_{2}=0.95$ and $q_{3}=0.98$, unstable point $S_{0}$ has been stabilized, as shown in Figure 1, note that $u_{1}=-y z, u_{2}=-z x, u_{3}=x y-\mu z$. The control is activated when $t \geq 20$, and the evolution of $x(t), y(t), z(t)$ is chaotic, then when the control is started at $t=22.5$ we see that $S_{0}$ is rapidly stabilized.

For $q_{1}=0.93, q_{2}=0.95$ and $q_{3}=0,98$, the unstable point $S_{1}$ has been stabilized, as shown in Figure 2.

For $q_{1}=0.92$ and $q_{2}=q_{3}=0,97$, the unstable point $S_{2}$ has been stabilized, as shown in Figure 3.

For $q_{1}=q_{2}=q_{3}=0,94$, the unstable point $S_{3}$ has been stabilized, as shown in Figure 4.

For $q_{1}=q_{2}=q_{3}=0,96$, the unstable point $S_{4}$ has been stabilized, as shown in Figure 5.

When $t$ is less than 20 , there is a chaotic behavior,

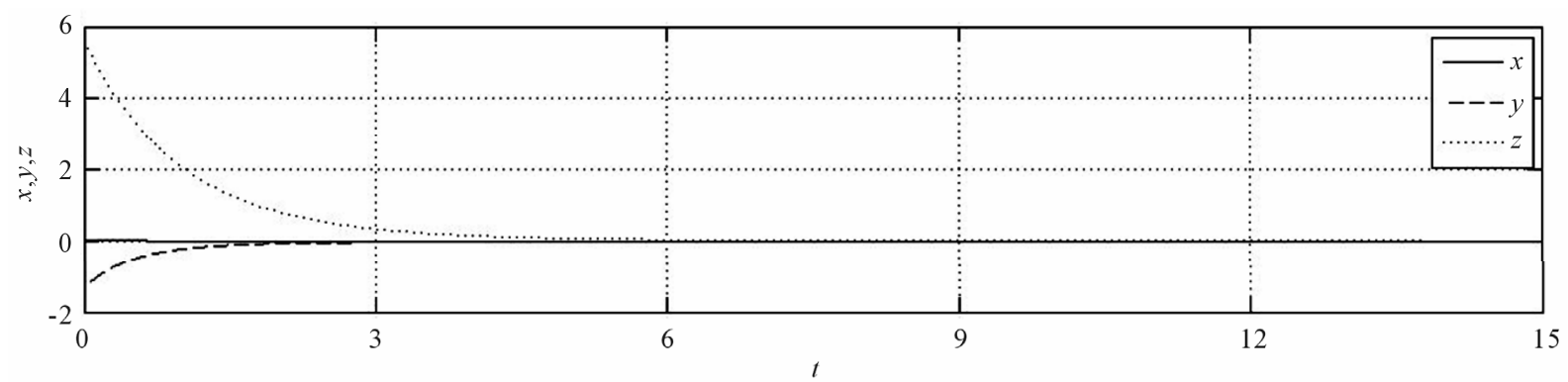

Figure 1. Stabilizing the equilibrium point $S_{0}$ for $q_{1}=0.93, q_{2}=0.95$ and $q_{3}=0.98$.

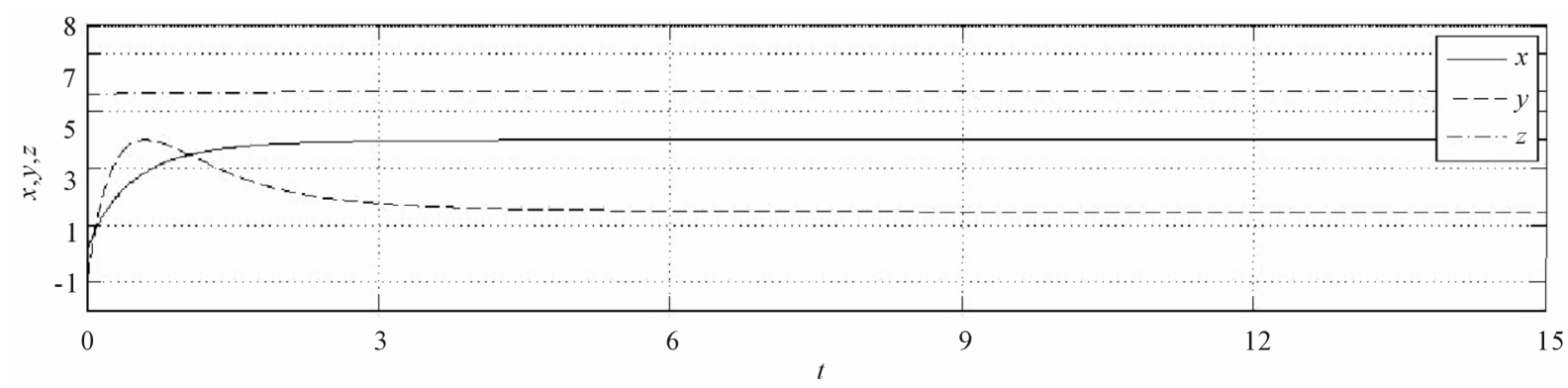

Figure 2. Stabilizing the equilibrium point $S_{1}$ for $q_{1}=0.93, q_{2}=0.95$ and $q_{3}=0.98$.

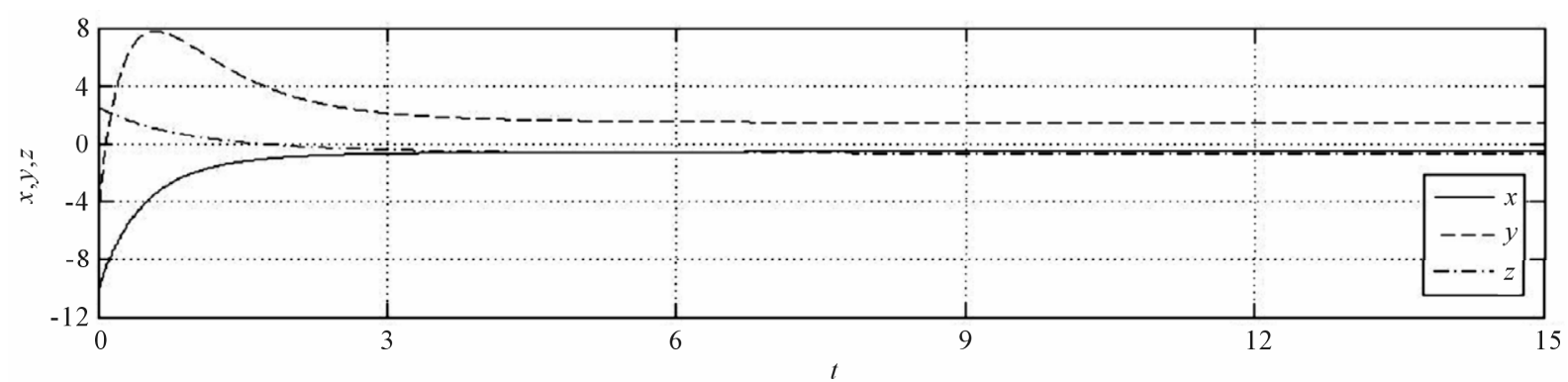

Figure 3. Stabilizing the equilibrium point $S_{2}$ for $q_{1}=0.92$ and $q_{2}=q_{3}=0.97$. 


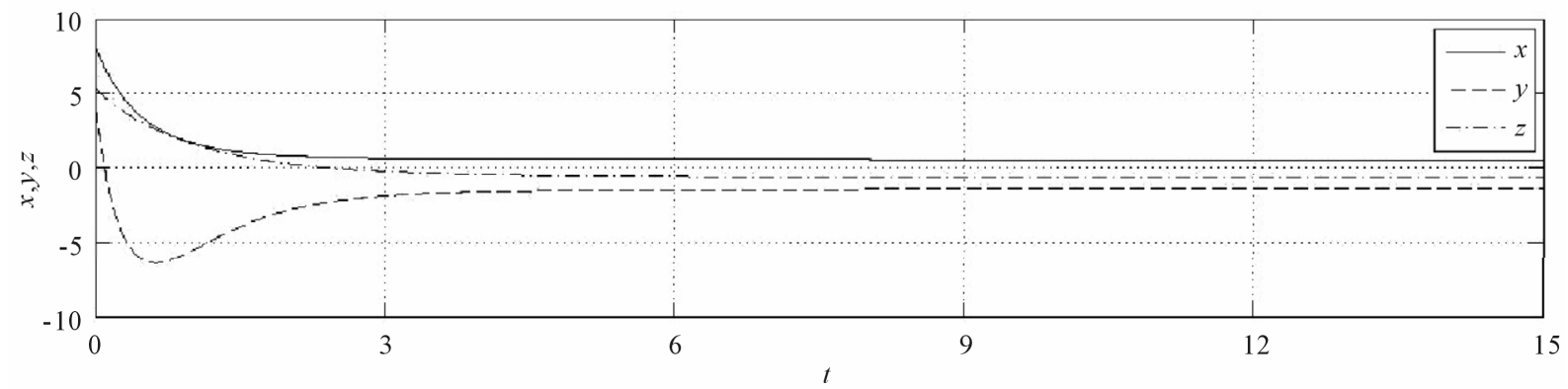

Figure 4. Stabilizing the equilibrium point $S_{3}$ for $q_{1}=q_{2}=q_{3}=0.94$.

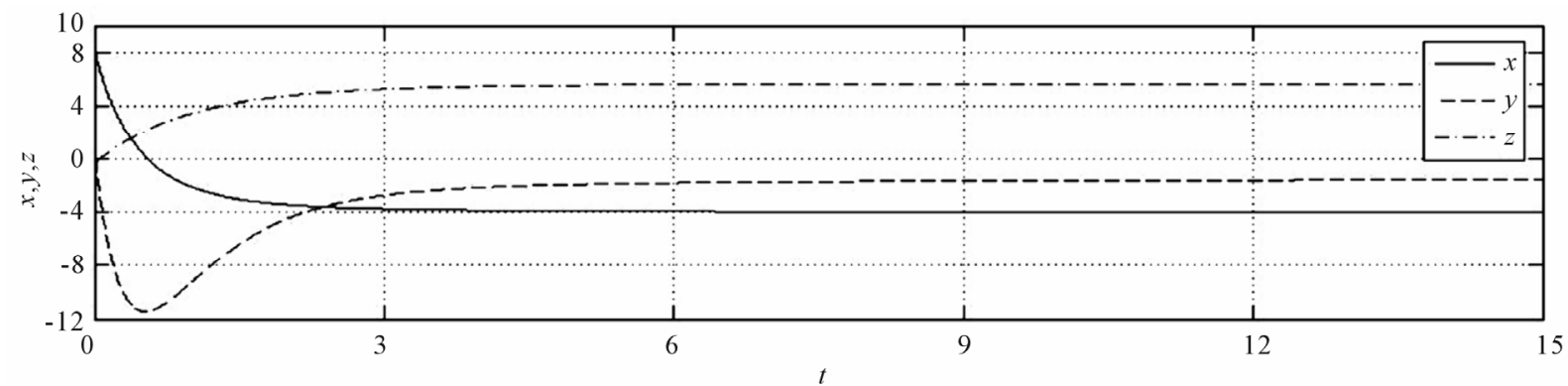

Figure 5. Stabilizing the equilibrium point $S_{4}$ for $q_{1}=q_{2}=q_{3}=0.96$.

but when the control is activated at $t=20$, the five points $S_{0}, S_{1}, S_{2}, S_{3}$ and $S_{4}$ are rapidly stabilized.

\section{Conclusions}

Chaotic phenomenon makes prediction impossible in the real world; then the deletion of this phenomenon from fractional order system is very useful, the main contribution of this paper is to this end.

In this paper, we investigate the system with fractional order applying the fractional calculus technique. According to the stability theory of the fractional order system, dynamical behaviors of the fractional order system are analyzed, both theoretically and numerically. Furthermore, nonlinear feedback control scheme has been extended to control fractional order system. The results are proved analytically by stability condition for fractional order system. Numerically the unstable fixed points have been successively stabilized for different values of $q_{1}, q_{2}$ and $q_{3}$. Numerical results have verified the effectiveness of the proposed scheme.

\section{Acknowledgements}

This work is supported by the Qing Lan Project of Jiangsu Province under the Grant Nos. 2010 and the 333 Project of Jiangsu Province under the Grant Nos. 2011.

\section{REFERENCES}

[1] R. C. Koeller, "Application of Fractional Calculus to the
Theory of Viscoelasticity," Journal of Applie, Vol. 51, No. 2, 1984, pp. 299-307. doi:10.1115/1.3167616

[2] H. H. Sun, A. A. Abdelwahad and B. Onaral, "Linear Approximation of Transfer Function with a Pole of Fractional Order," IEEE Transactions on Automatic Control, Vol. 29, No. 5, 1984, pp. 441-444. doi:10.1109/TAC.1984.1103551

[3] M. Ichise, Y. Nagayanagi and T. Kojima, "An Analog Simulation of Noninteger Order Transfer Functions for Analysis of Electrode Process," Journal of Electroanalytical Chemistry and Interfacial Electrochemistry, Vol. 33, No. 2, 1971, pp. 253-265. doi:10.1016/S0022-0728(71)80115-8

[4] O. Heaviside, "Electromagnetic Theory," Chelsea, New York, 1971.

[5] T. T. Hartley, C. F. Lorenzo and H. K. Qammer, "Chaos in a Fractional Order Chua's System," IEEE Transactions on Circuits and Systems I, Vol. 42, No. 8, 1995, pp. 485490.

[6] I. Grigorenko and E. Grigorenko, "Chaotic Dynamics of the Fractional Lorenz System," Physical Review Letters, Vol. 91, No. 3, 2003, Article ID: 034101. doi:10.1103/PhysRevLett.91.034101

[7] C. Li and G. Chen, "Chaos in the Fractional Order Chen System and Its Control," Chaos, Solitons and Fractals, Vol. 22, No. 3, 2004, pp. 549-554. doi:10.1016/j.chaos.2004.02.035

[8] C. P. Li and G. J. Peng, "Chaos in Chen's System with a Fractional Order," Chaos, Solitons and Fractals, Vol. 22, No. 2, 2004, pp. 443-450. doi:10.1016/j.chaos.2004.02.013

[9] Z. M. Ge and A. R. Zhang, "Chaos in a Modified van der 
Pol System and in Its Fractional Order Systems," Chaos, Solitons and Fractals, Vol. 35, No. 2, 2007, pp. 17911822 . doi:10.1016/j.chaos.2005.12.024

[10] C. G. Li and G. R. Chen, "Chaos and Hyperchaos in Fractional Order Rossler Equation," Physica A, Vol. 341, No. 10, 2004, pp. 55-61. doi:10.1016/j.physa.2004.04.113

[11] W. Zhang, S. Zhou, H. Li and H. Zhu, "Chaos in a Fractional-Order Rossler System," Chaos, Solitons and Fractals, Vol. 42, No. 3, 2009, pp. 1684-1691. doi:10.1016/j.chaos.2009.03.069

[12] D. G. Varsha and B. Sachin, "Chaos in Fractional Ordered Liu System," Computers \& Mathematics with Applications, Vol. 59, No. 3, 2010, pp. 1117-1127. doi:10.1016/j.camwa.2009.07.003

[13] M. Caputo, "Linear Models of Dissipation Whose Q Is Almost Frequency Independent-II," Geophysical Journal of the Royal Astronomical Society, Vol. 13, No. 5, 1967, pp. 529-539. doi:10.1111/j.1365-246X.1967.tb02303.x

[14] J. W. Xiang and W. Hui, "A New Chaotic System with Fractional Order and Its Projective Synchronization," Nonlinear Dynamic, Vol. 61, No. 3, 2010, pp. 407-417. doi:10.1007/s11071-010-9658-x

[15] K. Diethelm, N. J. Ford and A. D. Freed, "A Predictor-Corrector Approach for the Numerical Solution," Nonlinear Dynamic, Vol. 29, No. 1-4, 2002, pp. 3-22. doi:10.1023/A:1016592219341 